\title{
Pensar en tiempo de Revolución: antología esencial
}

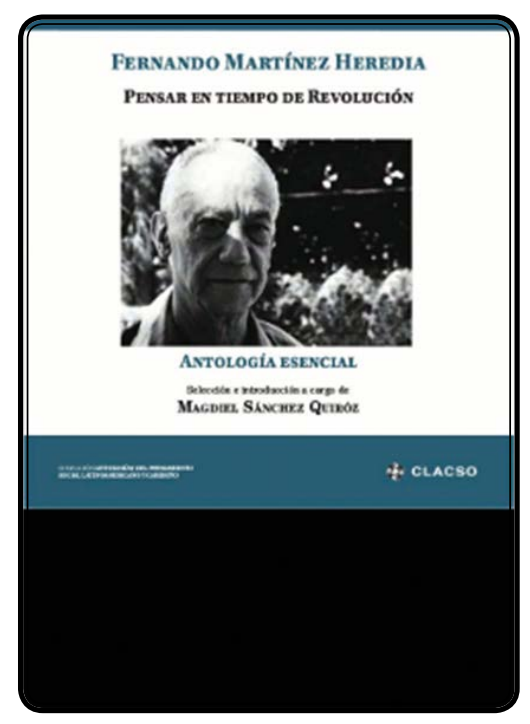

Pensar en tiempo de Revolución: antología esencial / Fernando Martínez Heredia. 2018. compilado por Magdiel Sánchez Quiróz. Ciudad Autónoma de Buenos Aires: CLACSO

Pensar significa traspasar Ernst Bloch

\section{I}

¿Cómo un ser humano común se convierte en extraordinario? ¿Qué necesita o qué posibilita que un ser humano vaya más allá de lo que lo su entorno social le permite? ¿Cómo un pensamiento puede trascender el horizonte teórico de su época?

¿Cuándo Fernando Ramón, un humilde joven nacido en Yaguajay se convierte en el Fernando Martínez Heredia reconocido en Cuba? Podría responderse rápidamente que al recibir el premio de ensayo Casa de las Américas en 1989.

O cuando apareció por primera vez su nombre como director de la revista Pensamiento Crítico. Quizás al ingresar al Departamento de Filosofía de la Universidad de La Habana. ¿Fue en sus primeras batallas teóricas cuando entró a la escuela destinada a formar profesores de Filosofía? Al ahondar en su vida encuentro que antes ya había tenido sus primeros choques teóricos y que podría pensarse en aquella vez que un joven, tras el triunfo de la Revolución, se acercó a él para invitarlo a alzarse contra Fidel Castro, "porque Fidel quería hacer a Cuba comunista" y él se negó y pensó: "Si Fidel es comunista, entonces yo también soy comunista". Y cuando como combatiente del Movimiento 26 de Julio del Frente Norte de Las Villas escribió un manifiesto en que hablaba de que la revolución se hizo para acabar con 400 años de explotación del hombre por el hombre. Mejor habría que remontarse a la guerra, a la batalla contra la dictadura, a los combates en los momentos en que las ideas y las armas evidencian una realidad distinta a la que aparece en la academia. ¿O bien sería cuando de joven se enteró de que un grupo de rebeldes se había alzado contra la dictadura y habían sido masacrados y entonces anotó en su libreta los nombres de los asesinados que los medios iban dando a conocer, como para que no se perdiera la memoria de los caídos en un cuartel de Santiago, el Moncada?

La vida de Fernando Martínez Heredia (FMH, en adelante) se inscribe en el proceso revolucionario que vivió por más de 60 años. Años y vivencias que para las ciencias sociales suelen explicarse como excepciones o accidentes, por ser difícilmente asibles a sus métodos de 
análisis, pero que desde los actores que alteraron la historia se expresa como un esfuerzo -con dimensiones individuales y colectivas más allá de su horizonte nacional, pero bien arraigado a este- por romper con las determinaciones de lo factible. Pasar por encima de lo permisible y hacedero en el ámbito de la reproducción de la vida social. Quebrar las determinaciones de la geopolítica $\mathrm{y}$, en suma, del horizonte histórico de una época y, al hacerlo, echar por la borda las leyes de la ciencia positiva (aun en sus presentaciones críticas), de la determinación de la política, del ser social y su conciencia social por la economía. En suma: romper los límites de lo posible (expresión recurrente de FMH, síntesis de vida y esfuerzos teóricos, y de la herejía cubana que se desató con todas sus fuerzas un primero de enero de 1959).

Desde Cuba, a partir de sus vivencias, estudio y del enorme cúmulo de experiencias y relaciones nacidas de la revolución, Fernando fue formulando un pensamiento que encontró su planteamiento más acabado en el concepto de transición socialista y en un amplio conjunto de elementos que lo conforman y con los que se relaciona.

Elaboración y desarrollo propio, en el marco de un movimiento socialista más amplio, pero con raíz y fundamento primordial en el suelo cubano. Un pensamiento en tiempo de revolución que, visto en su conjunto y en relación con la praxis de quién lo elaboró, muestra notables diferencias con otras expresiones críticas de la época. A partir de su raíz cubana y en relación con el pensamiento crítico marxista, asumió como desafío pensar cómo acabar con todas las dominaciones. Tarea que, tenía claro, implicaba romper con los límites de lo posible, pues solo a partir de la violentación del horizonte de posibilidad establecido por el pensamiento y la sociedad dominantes se puede actuar históricamente, abriendo nuevas sendas en el pensamiento, la acción y más aún, en la vida de los seres humanos. La presente antología da cuenta, en un material de amplia extensión, de la trascendencia de FMH, la originalidad de un pensamiento y del conjunto de temas que desarrolló de acuerdo a circunstancias disímiles.

\section{II}

En el poblado de Yaguajay, región azucarera del centro de Cuba, nace FMH el 21 de enero de 1939. El contexto es el de la Segunda República neocolonial burguesa configurada para impedir que ocurriera otra revolución en la corta vida de esa nación: ya había vivido un largo período de guerras de independencia (1868-1878 y 18951898) y recientemente una revolución en los años treinta que había echado abajo a la Primera República. Su entorno familiar está conformado por una identidad negra, independentista $y$ patriota, entramado que se alimenta de los relatos latentes de los sobrevivientes de la guerra de independencia. Entre las consignas que le imprimirán un valor central a su carácter revolucionario está el lema: "Vergüenza contra dinero" formulado por Eduardo Chibás, líder del Partido Ortodoxo, agrupación de masas de enorme arrastre popular opuesta a la corrupción reinante en la República. Desde joven, su pasión por la historia y la lectura lo van moldeando de una particular manera y los sucesos que pudiesen parecer marginales, como el asalto a un par de cuarteles por un grupo de jóvenes que enfrentaba al dictador, resuenan en él y lo motivan llevar el registro de sus nombres.

En ese recordar y apropiarse del pasado rebelde, se va identificando con personajes como Julio Antonio Mella y Antonio Guiteras Holmes, quienes fuera de la iconografía oficial nutrían el imaginario radical de la época. Ellos, junto a José Martí, se convertirán en sus referentes morales y de acción a lo largo de toda su vida.

\section{El surgimiento del Movimiento 26 de Julio como un lugar para pelear...y pensar}

El legado de Mella y Guiteras que FMH hace propio, lo lleva a incorporarse al Movimiento 26 de Julio antes que Fidel Castro y los suyos desembarcaran en la Isla. Es la convicción profunda en la necesidad de una transformación lo que lo lleva a tomar esa decisión siendo muy joven.

Constituye parte de ese gran movimiento que avanzaba por el fuerte liderazgo de Fidel, el 
programa contenido en La historia me absolverá (Castro Ruz, 2001 [1953]), la idea de violencia revolucionaria y la insurrección como necesarias y determinantes del cambio. Un socialismo, que aun sin presentarse como tal, se hacía de una tradición de casi 100 años de lucha en ese país. FMH desarrolló labores de combatiente en Las Villas, es testigo de la Batalla de Santa Clara, donde verá al comandante Ernesto Che Guevara dirigir los combates. Con el triunfo seguirá involucrado en tareas revolucionarias, apresurará la soñada reforma agraria en la región de Las Villas y asumirá como un deber propio pensar en la revolución y desarrollar capacidades para hacer que el proceso no se detenga y fracase. Sus ansias de conocimiento lo llevan a ingresar a la Universidad de La Habana, donde pretendía estudiar Filosofía, pero finalmente optó por Derecho. Librará batallas en la facultad y diversos espacios. Asumirá las tareas de enseñanza masiva, como las del Plan Fidel de educación ${ }^{1}$.

\section{El Departamento de Filosofía hasta el 71, el espacio para profundizar la revolución}

Dice FMH: "la Filosofía me emboscó, en el mejor estilo de entonces, me cambió el FAL por un Manual de Konstantinov, y eso dio lugar a una década de combates intelectuales" (Martínez Heredia, 2010b: 73). Fue en medio de una gran efervescencia social, luego de la declaración del carácter socialista de la revolución cubana, el 16 de abril de 1961, que los cubanos se abalanzaron sobre todo lo que fuera marxismo. Como parte de la Reforma Universitaria de 1962, que convertía en obligatoria la impartición de la enseñanza de la Filosofía y Economía Política marxista en todas las carreras universitarias de la Universidad de La Habana, la Universidad Central y la
Universidad de Oriente, participará en un curso para conformar el Departamento de Filosofía, encargado de llevar a cabo esa tarea.

En la escuela, chocó abiertamente con las versiones oficiales, al tiempo que comienza a liderar -al interior de aquel grupo de jóvenes en formación- una posición común que desafía el dogma y busca construir un pensamiento radical y original.

El grupo de la Calle K, como será conocido por la ubicación de sus oficinas, trabajará muy cercano a Fidel Castro, quien los visita con frecuencia y promueve el debate. Alentados por él y el entonces presidente Osvaldo Dorticós, se harán cargo de las Ediciones Revolucionarias, publicarán la revista Pensamiento Crítico y harán tareas de intercambio, formación y divulgación con destacados intelectuales de diversos países, con revistas de la época y con revolucionarios del mundo. En una reunión, el Presidente Dorticós les asigna una tarea tan ambiciosa como aparentemente imposible: incendiar el océano $^{2}$. Asumiendo el desafío, que implicaba a su vez una tarea de interpretación de aquel encargo, el grupo le imprimió su propio sello: "Hay que hacer que el 'marxismo-leninismo' se ponga a la altura de la Revolución Cubana" (Martínez Heredia, 2010a: 91).

Es entonces cuando, desde el Departamento de Filosofía, FMH se pone al frente de una de las polémicas más serias en torno al desarrollo del socialismo en la isla. Es Cuba en los años sesenta: etapa de profundización del socialismo, en la que la posición autóctona y la soviética se disputaban cómo controlar la economía y el poder de transición socialista.

1 En la sección VIII. Sobre su vida de esta antología se comparten textos que abundan en lo aquí relatado.

2 "Nosotros debemos conformar la enseñanza del marxismo-leninismo fundamentalmente -sin perder de vista, desde luego, la realidad universal-, por nuestra realidad histórico-social concreta, a la cubana. Y para eso no existe ningún manual [...]. Es un deber fundamental de ustedes procurar dar una enseñanza muy viva, muy vinculada a la realidad cubana, a la historia cubana [...]. Lo más importante es que ustedes enseñen a pensar a los alumnos, a crear en los alumnos la capacidad de pensar y de razonar por sí mismos, con un sentido crítico [...]. Yo les digo que hay que incendiar el Atlántico, ¡y ustedes miren a ver cómo lo incendian!" (Dorticós, 2013: 382 y ss.). 
Ernesto Che Guevara, como presidente del Banco Nacional y ministro de Industrias sostuvo una de las discusiones más trascendentales en el plano económico frente a Carlos Rafael Rodríguez y Charles Bettelheim. En ciertos análisis, los puntos de este debate se mostraron alrededor del tema de la autogestión o del Sistema Presupuestario de Financiamiento, pero el verdadero nudo de la cuestión giraba en torno a la ley del valor, la transición socialista y al papel de la conciencia y la subjetividad en la revolución. En materia de historia, Manuel Moreno Fraginals y Jorge Ibarra polemizaban con Sergio Aguirre y Julio Le Riverend en torno a la interpretación histórica del siglo XIX y sus relaciones con las tareas políticas del presente. En torno al arte, la literatura y las políticas culturales de fidelistas consumados como Alfredo Guevara, Tomás Gutiérrez Alea y Julio García Espinoza, a los que se les sumarán Ambrosio Fornet y Jesús Díaz, se enfrentó a militantes del Partido Socialista Popular como Blas Roca, el Indio Naborí, Mirta Aguirre (directora del Consejo Nacional de Cultura) y Edith García Buchaca que trataron de imponer la política cultural dictada desde la Unión Soviética y que ya se había esbozado desde los tiempos de la dictadura de Batista con la "Conversación con nuestros pintores abstractos" de Juan Marinello. En el plano intelectual, la pelea será entre el socialismo cubano, promovido por el Departamento de Filosofía, y el socialismo de matriz soviética que representaban los responsables de las Escuelas de Instrucción Revolucionarias (EIR), provenientes del viejo Partido Socialista Popular. FMH, como principal responsable del Departamento, captó en su mejor expresión el centro de la polémica. Más que virtudes excepcionales (indudablemente presentes), era la experiencia combativa vivida en primera persona lo que le imprimía un carácter especialmente incisivo a la comprensión cabal y profunda de los problemas que se avecinaban. Este es un punto central, puesto que la mayoría de los jóvenes que formaban parte del Departamento (FMH era el mayor de todos con solo 26 años), no habían tenido la oportunidad de participar como combatientes en la revolución. FMH había iniciado su militancia a los 15 años, mientras otros miembros del Departamento eran apenas niños. Lo más frecuente es recordar al Grupo de la Calle $\mathrm{K}$ en relación con los debates sobre los manuales soviéticos, el cierre del Departamento y la censura de su revista, Pensamiento Crítico. Sin embargo, lo que no se toma en cuenta es la polémica más de fondo, en la que todos aquellos elementos se encontraban subordinados al problema de los fundamentos humanos y teóricos para la transición socialista. Y allí es donde el Departamento y la posición de Fidel Castro se convertían en una sola. Sin que necesariamente existiera una fina coordinación, ni que aquel grupo se convirtiera en "los intelectuales de Fidel", ellos asumieron esta pelea desde una posición autónoma, rebelde y radical. Al respecto puntualizó FMH: "Son discusiones por el poder, no solamente de ideas. De dos facciones de la revolución en el poder" (Martínez Heredia, 2016).

En esta época, FMH iniciará tareas internacionalistas fundamentales para el proceso revolucionario (solamente algunas relacionadas con el Departamento), lo que se transformó en un momento decisivo de su formación. Conocerá a combatientes de otros países, y como parte del esfuerzo por desatar procesos revolucionarios en otras partes del mundo, en especial del continente, se relacionará con los procesos de Chile, Perú, Nicaragua, Venezuela El Salvador. En ese transcurso, convivirá con teóricos reconocidos y debatirá con ellos, enriqueciendo su posición política y filosófica revolucionaria.

Los textos más relevantes de esa época son "El ejercicio de pensar", "Marx y el origen del marxismo" y sus ponencias para el Congreso Cultural de La Habana (1968) y el Congreso de Educación y Cultura (1971) -todos incluidos en la presente antología. Además, la labor del Departamento quedará registrada tanto en la memoria de esa nueva generación universitaria que llenó de pueblo la Universidad -en palabras del Che- como con las dos ediciones de las Lecturas de Filosofía (dos tomos) y de los 53 números de la revista Pensamiento Crítico, que 
de 1967 a 1971 se publicó mensualmente con tirajes de hasta 15 mil ejemplares ${ }^{3}$.

\section{El ostracismo y el desafío revolucionario}

Al ver los cohetes que tiraron en casa de Mario, me he jurado que los americanos van a pagar bien caro lo que están haciendo. Cuando esta guerra se acabe, empezará para mí una guerra mucho más larga y grande: la guerra que voy a echar contra ellos. Me doy cuenta que ese va a ser mi destino verdadero (Castro, 1958). Estas conocidas palabras de Fidel Castro, contenidas en una carta a Celia Sánchez, se suelen citar como la clara referencia al momento en que Fidel se perfiló como un acérrimo enemigo del imperialismo. La sentencia queda marcada sobre todo en la heroica resistencia contra el bloqueo y los múltiples ataques que el pueblo cubano resistió bajo su mandato. Pero el sentido profundo de esa aseveración no se limita a la oposición al imperialismo, sino que es, sobre todo, la apuesta que movilizó a Fidel en la vida entera: hacer una revolución en todo el mundo. Y desde Cuba persiguió desde todos los frentes este objetivo. Existía un entendimiento cabal de Fidel al respecto: la única posibilidad de sostener y profundizar la revolución estaba estrechamente vinculada con su expansión en todo el mundo y en especial en América Latina. De ello se desprende que Cuba haya encomendado a uno de sus mejores hombres a la tarea de expandir la revolución. Aunque los relatos dominantes lo ubiquen como un aventurero empedernido, Ernesto Che Guevara era en realidad un servidor de la revolución, cubana en primer lugar y latinoamericana en consecuencia, que dejó todas sus tareas al interior de la isla para abrir nuevos desafíos internacionalistas.

El surgimiento de la Organización para la Solidaridad con los Pueblos de África, Asia y América Latina (OSPAAL), la Organización Latinoamericana de Solidaridad (OLAS) y la
Conferencia Tricontinental son algunos de los esfuerzos visibles que demuestran el empeño que se invirtió en lograr la irradiación revolucionaria. Pero no se logró. A la caída en combate del Che le sucedió un momento muy difícil en materia económica al verse frustrado el plan económico que suponía -entre otras medidas- alcanzar una zafra de 10 millones de toneladas para el año 70, para así lograr un crecimiento económico que rompería con la dependencia económica. El plan fracasó y Cuba se vio forzada a entrar en una relación de subordinación con la Unión de Repúblicas Soviéticas Socialistas (URSS) -que, a pesar de la cercanía, siempre estuvo permeada de tensiones- a través del Consejo de Ayuda Mutua Económica (CAME). Esto último provocó una mayor cesión de espacios de poder a la fracción cercana a la URSS. La unidad revolucionaria al interior de Cuba, una de las mejores construcciones de Fidel, se volvió más difícil, compleja y necesaria. Las fuerzas locales identificadas y relacionadas con la URSS ganaron peso. Sin dejar los espacios de poder básicos para el sostenimiento soberano de la Revolución, el bloque liderado por Fidel cedió más espacios en el poder a las fuerzas que provenían del Partido Socialista Popular. Se apoderaron de la cultura y la enseñanza, sin que por ello el proyecto revolucionario se modificara en lo sustancial. Las nuevas circunstancias fueron aprovechadas por algunos para imponer la censura, cancelar los desafíos culturales abiertos, en parte, por el Departamento de Filosofía.

El Departamento fue cerrado, la revista Pensamiento Crítico también y el grupo de la Calle $\mathrm{K}$ disuelto. Meses después fue removido el rector de la Universidad de La Habana, José Miyar Barrueco, hombre cercano a Fidel, y con ello se cerró una etapa en que la crítica y el poder libertario inundaban la Universidad y las ciencias. La censura dominó el arte, las ciencias, la música, el teatro y todos los ámbitos culturales. FMH, más que otros miembros

3 La historia del Departamento es sumamente apasionante y rebasa las dimensiones de esta Presentación. El lector podrá encontrar en los textos "A cuarenta años de Pensamiento Crítico", "Cuba y el pensamiento crítico" y "Conversación sobre los años sesenta" textos al respecto. Además, esta antología incluye dos presentaciones de la revista Pensamiento Crítico, la del N 1 y la del 39, escritas por FMH $(1967,1970)$. 
del Departamento, fue aislado de las tareas intelectuales.

Lejos de las aulas y de los centros de estudio, padeció una censura casi absoluta y tuvo que continuar sus labores intelectuales por cuenta propia en un terreno lleno de dificultades. Fiel a la Revolución, continuó trabajando, aunque fuera de foco, sosteniéndose de manera ejemplar en un periodo que él mismo nombró ostracismo.

Siguió su labor abordando los temas que más le apasionaban, como las tensiones entre los ideales y la racionalidad en cada una de las revoluciones de Cuba, el estudio de la revolución del 30 -en especial sobre Antonio Guiteras-, sus trabajos sobre la Revolución de 1959 y una historia social de su natal Yaguajay. Contradictoriamente, es en esos años donde, a pesar de su exclusión, sigue asumiendo tareas internacionalistas que lo llevan a varios países de la región. Vale señalar que nunca usó este oscuro momento, para lograr algún beneficio personal o alcanzar la fama "disidente", figura que seguramente le hubiera garantizado una vida "destacada" en el mundo intelectual del establishment mundial. De esto da cuenta su discreción con respecto a esta etapa de su vida, que es quizás en la que con mayor fuerza maduró en su personalidad militante. Como veremos, su actitud tuvo más que ver con su claridad política en relación a su apuesta por la revolución, que con una forma de sometimiento silencioso.

Su trabajo continuó siendo crítico y abonando a la construcción de la unidad revolucionaria de su país y de América Latina, priorizándola por sobre aspiraciones personales, académicas y de distinción sobre el resto.

\section{Los ochenta, el premio Casa y los noventa}

En 1976 ingresó al Centro de Estudios sobre Europa Occidental, donde vuelve a desempeñar actividades intelectuales. Tras el triunfo sandinista en Nicaragua en 1979, viaja a ese país para asumir tareas diversas de apoyo a la revolución en ese país. En 1984 regresa a Cuba para trabajar en el Centro de Estudios de América (CEA), donde se reencuentra con viejos compañeros del Departamento como Hugo Azcuy y Aurelio Alonso. Son los años previos a que los regímenes socialistas de Europa oriental sucumban desde dentro, por obra de sus direcciones. Cuba, a contracorriente de esa ola, a través de Fidel Castro empuja el Periodo de Rectificación de Errores y Tendencias Negativas, que va a tener como inspiración central a Ernesto Che Guevara. Es un esfuerzo por evitar que Cuba sea arrastrada por los países socialistas, y, al mismo tiempo, por la radicalización del proceso y por desmontar la influencia soviética en torno a la política interna, hegemónica durante los años previos. El grupo del CEA se dedica a estudiar al Che, y FMH participa activamente en esta tarea. También escribe y publica, enfrentando grandes adversidades, su primer libro, Desafíos del socialismo cubano (Martínez Heredia, 1988), donde expone, por primera vez, su concepto de transición socialista.

Pero FMH vuelve al ruedo con mayor fuerza cuando recibe el Premio Extraordinario de Ensayo de Casa de las Américas en 1987 por su libro Che, el socialismo y el comunismo (Martínez Heredia, 1989). Gracias a ese reconocimiento vendrá una nueva fase de combates públicos que lo colocan en el centro del ambiente intelectual cubano. Vuelve a enfrentarse a adversidades y reiteradas censuras de las que no logra escapar a pesar del prestigio. Serán también momentos de amplia articulación con movimientos populares y organizaciones en América Latina e incluso, a pesar de ya no ser un joven, sigue participando en tareas de defensa de la patria. Sus labores de articulación política crecen con Venezuela, Argentina, Brasil y los movimientos sociales más reconocidos del continente. Desde fines de los ochenta acompañará, como miembro fundador, al Centro Memorial Martin Luther King Jr., un espacio ecuménico de revolucionarios cubanos que desde el ámbito de la sociedad civil asumen su ejercicio político dando diversas batallas por el socialismo, contra la visión ateísta $\mathrm{y}$ promoviendo el trabajo de base y de educación popular.

En 1996 se ordena el cierre del CEA por falsas acusaciones en contra de sus miembros, que rememoran los ataques que sufrieron los 
mismos integrantes en los años setenta en el Departamento de Filosofía. El suceso desató una gran crisis. La Unión de Escritores y Artistas Cubanos (UNEAC) presentó una protesta.

Se crearon comisiones en los Centros de Investigación y se abrió un proceso que fue conocido como el Caso CEA y que, a través de unas filtraciones, fue convertido en una publicación para condenar a la dictadura cubana (Giulano,1998). Por intervención directa de Armando Hart, entonces ministro de Cultura, FMH comenzó, en septiembre de ese mismo año, a trabajar en el Centro de Investigación y Desarrollo de la Cultura Cubana Juan Marinello, que en 2007 cambiará de nombre a Instituto Cubano de Investigación Cultural Juan Marinello, y donde desarrollará una intensa labor de investigación hasta el final de su vida. En contraposición con los ataques, recibe ese mismo año la Orden por la Cultura Nacional 1996.

Por esos años trabajará intensamente en América Latina. Dará numerosas entrevistas y conferencias y será parte del comité editorial de la revista América Libre. En 2001 por primera vez logra publicar un libro en Cuba que no sufrirá contratiempos ni censuras, El corrimiento hacia el rojo (Martínez Heredia, 2001a) por la Editorial Letras Cubanas. Nuevas generaciones descubren a través del libro a un pensador desconocido, del que brotan reflexiones sumamente interesantes y ricas que les permiten una identificación original con el proceso revolucionario.

\section{La última etapa, nuevos combates ante nuevos escenarios}

De 2007 hasta su muerte será la etapa más prolífica de su producción, al menos en el ámbito de la difusión y la publicación de materiales. El uso de las herramientas digitales será un nuevo vehículo que le permitirá fortalecer su incidencia en nuevas generaciones. Las usa y aprovecha defendiendo también el uso y producción de libros. En 2007 recibe el Premio Nacional de Ciencias Sociales, la máxima distinción para un pensador social en Cuba. Son nuevos tiempos en que sus publicaciones empiezan a ser más conocidas y se difunden en todo el país. Se cumplen 40 años de la publicación del primer número de la revista Pensamiento Crítico, lo que motiva su rescate y que la experiencia en torno a ella y al Departamento se estudie. Desarrolló cursos con jóvenes, en especial a través de la cátedra Gramsci del Marinello, pero también fomentó muchos espacios fuera de la academia. Dejó un abanico de proyectos pendientes, los cuales, sin duda, serían sumamente fructíferos para el pensamiento social, sobre todo en momentos tan difíciles como el presente. Nunca se jubiló, trabajó hasta las últimas horas de vida. Combatió hasta el final con la misma voluntad.

En los últimos años de su vida libró una de sus batallas más importantes, con plena conciencia del propósito, pero quizás desconociendo los impactos que podría tener: construir una visión propia, de Cuba, de sus problemas actuales, y en función de ellos, de su perspectiva histórica y los desafíos para la Revolución cubana. Cuestionado por su optimismo, FMH confiaba en las fuerzas sociales, en el acumulado cultural del país, para seguir dando lecciones históricas. Ubicaba el conflicto del momento entre avanzar en una vía revolucionaria o transitar a una posrevolución. Y alertaba que en caso de que avanzara la segunda opción estallaría una guerra civil. La fuerza del pueblo sería, pues, la definitoria en los combates por venir, en los que Martí y Fidel tendrían que liderar de nueva cuenta esa batalla.

$\mathrm{Su}$ inquietante pensar también lo mantuvo concentrado en problemas teóricos de la historia y el análisis histórico, el pensamiento de Karl Marx y América Latina, región suya en la que sostuvo intercambios y reflexiones, territorio de esperanza libertaria y espacio privilegiado para que su pensamiento florezca, no solo en los ámbitos del saber, las universidades y la academia, sino sobre todo en las calles, los pueblos, las luchas y las revoluciones futuras, las pendientes.

\section{III}

\section{Contenidos de la antología, los temas del autor}

Pensar en tiempo de revolución es una forma sintética de referirse a la vida y la obra de FMH. 
El recorrido general sobre su vida nos permitió mostrar las formas en que el ejercicio del pensar de un militante revolucionario y trabajador intelectual se van desarrollando y alterando por el decurso y los desafíos de la práctica revolucionaria galvanizada con el proceso histórico de su país.

La antología se ha estructurado en nueve secciones guiadas por la idea de que la producción teórica de FMH es pensar en tiempo de revolución. A continuación, hacemos una presentación panorámica del contenido de cada una de esas secciones.

\section{Sección I. Sobre la teoría y el pensamiento}

$\mathrm{FMH}$ fue un revolucionario al que -como mencionamos antes- la filosofía lo emboscó, y él, en respuesta, cimbró el pensamiento revolucionario en y para su país. Desde sus inicios como revolucionario dedicado al trabajo intelectual se empeñó en reflexionar sobre las relaciones entre la práctica y la teoría, poniendo especial atención a las relaciones entre las ideologías, los pensamientos y la historia.

Esta primera sección se subdivide en tres apartados en función de los grandes problemas queFMHabordó comouna unidad, pero sobrelos cuales tuvo acentos específicos, de acuerdo a las polémicas en que participó porque, en palabras de su compañero Germán Sánchez Otero, "fue siempre un polemista" (Sánchez Otero, 2016). El nombre del primer apartado, Pensamiento social, define, de entrada, una concepción que en contraposición a las definiciones estrechas de las ciencias sociales, comprende las concepciones más generales, los modos de emprender su conocimiento y sus normas, con conceptos previos y fronteras, las pertenencias ideológicas de quienes los formulan y su vinculación con cuerpos epistemológicos más precisos ${ }^{4}$. Comprender el papel del pensamiento social en el proceso histórico resulta relevante para un pensamiento crítico y militante, que tiene que trabajar con los materiales humanos difíciles de asir y encasillar en los cajones que las ciencias sociales predisponen para ellos en función de la praxis reiterativa de la sociedad.

El primer ensayo que publicó abre esta antología y fue punta de lanza de una de las más intensas polémicas de la revolución: se titula "El ejercicio del pensar". Los textos "Pensamiento social y política de la Revolución" y las "Palabras al recibir el premio nacional de Ciencias Sociales" profundizan más sobre las relaciones entre pensamiento social, ciencias sociales y prácticas sociales. "Educación, cultura y revolución socialista" es un texto sumamente relevante que tuvo que esperar más de 30 años para ser publicado, ya que fue censurado cuando FMH lo presentó como ponencia en el Primer Congreso de Educación y Cultura, celebrado en La Habana en abril de 1971. En él, hace una crítica de las relaciones entre educación y cultura en la sociedad en revolución hacia el comunismo $\mathrm{y}$ de las formas latentes en que aún se expresan la cultura y educación burguesas. Insiste en que la toma del poder político es solo la premisa para revolucionar el conjunto de la vida social, tarea aún pendiente. Además, abunda en los problemas e implicaciones del quehacer intelectual en tanto no se ha roto el rol de elite que ocupa en el capitalismo y, en tanto adopta una forma colonizada del pensar, no logra hacer consciente la necesidad de superarlo.

Finalmente, la presentación del número uno de la revista Pensamiento Crítico nos presenta una especie de manifiesto sobre el quehacer intelectual y la revolución, una especie de compromiso político público que hará el autor para toda su trayectoria. La versión original es anónima, pero la redacción es de Fernando y es suscrita por el consejo de dirección, integrado, además, por Aurelio Alonso, Jesús Díaz, Ricardo Jorge Machado y Thalía Fung. El marxismo, en ese sentido, es un pensamiento social cuya historia se relaciona con la de las revoluciones en el siglo XX. Los dos primeros textos de esta segunda subsección -la "Presentación" al libro de textos del Departamento y su estudio sobre

4 Véase el texto "Pensamiento social y política de la revolución" en donde expone este concepto. 
"Marx y el origen del marxismo"- dan cuenta de ello. El texto sobre Karl Marx muestra una lectura profunda, erudita y no dogmática del autor: un estudio de los presupuestos ideológicos de Marx, de sus planteos en torno a la revolución y su contexto histórico. El texto sería el capítulo II de un primer tomo que nunca terminó de escribir, y que gracias a la labor actual de Esther Pérez, intelectual revolucionaria cubana y compañera de $\mathrm{FMH}$, podrá salir a la luz pronto.

FMH asumió la osadía de comprender y apropiarse del marxismo para Cuba. "En el cumpleaños de Lenin: Utopía y práctica política en El Estado y la Revolución", "Vida y Propuesta de Antonio Gramsci", "Gramsci en Cuba de los años sesenta" y "Problemas de la historia del pensamiento marxista: los tiempos de Mariátegui" son textos que -con referencias al contexto histórico, los problemas a los que los autores se enfrentaron y las formas originales en que intentaron resolverlos- logran colocar las posibilidades de su aprehensión desde la práctica revolucionaria cubana y sus posibles aportes al pensamiento marxista y revolucionario.

El texto que cierra esta subsección destaca aparentemente como disonante por tratar sobre Michel Foucault, un autor que se suele considerar antimarxista y al que se alude peyorativamente como posmoderno. La lectura de FMH sobre Foucault resalta el reconocimiento y la apropiación del marxismo por el filósofo francés a pesar de no hacerlo de modo explícito. También insiste en los desafíos que tiene para el pensamiento crítico socialista en la actualidad apropiarse de todo el conocimiento social y romper con las lecturas sesgadas y dogmáticas que se rehúsan a pensar más allá de lo establecido, aun cuando ello lleve el apelativo de revolucionario. Pensamiento social y marxismo convergen en la tercera subsección: "Pensamiento político". Fue este rubro en el que la reflexión del autor concretó su teoría en unidad con su práctica política incesante. En cada uno de los textos de esta subsección se pueden apreciar nociones originales y de una radicalidad que se oculta en el lenguaje sencillo -desarrollado para las condiciones en las que se produce, los espacios limitados para su difusión y los públicos diversos que lo reciben- para pensar la superación de las condiciones de dominación, posible solo a partir de la fusión de las ideas con la fuerza material que los pueblos en movimiento constituyen.

"Anticapitalismo y problemas de la hegemonía", texto que abre esta subsección, expone magistralmente una serie de problemas del accionar político como las relaciones entre poder y proyecto, organización, hegemonía, disputa cultural. El lector podrá encontrar un material radical y sugerente. La lectura crítica sobre el colonialismo, en especial los problemas que el pensamiento crítico tiene que enfrentar en ese rubro -en relación con la entera cultura de los países colonizados- es tratada en "El colonialismo en el mundo actual" y en "Revolución cubana contra los colonialismos y necesidad de Fanon", en los que muestra también el dominio de este autor.

"El carácter y la vía de la revolución" es un texto ligado a su concepción de socialismo en el que expone la posición insurreccional cubana y comparte rasgos de su relación con Miguel Enríquez, líder máximo del Movimiento de Izquierda Revolucionaria (MIR) de Chile. Las revoluciones en relación con las cuestiones sociales como la fe, la religión y el cristianismo fueron tratados por FMH desde la compleja realidad de los pueblos, de sus formas de resistencia contra la dominación y de aceptación de la misma, de las potencias que el corazón de un mundo sin corazón puede detonar como parte de un proceso revolucionario.

En “Cristianismo y Liberación ¿Revolución en el cristianismo?" además de exponer esas cuestiones de modo tan magistral que al ser leído por Leonardo Boff, este supuso que se encontraba frente a un gran teólogo, permite rastrear los contenidos de un debate que al interior de Cuba sostuvo contra el ateísmo como posición de Estado, y que apoyando el trabajo de la Red Ecuménica Fe por Cuba, el Centro Martin Luther King Jr. y la Iglesia Bautista Ebenezer de Marianao, entre otras agrupaciones, lograron un cambio en la política estatal que rompió con la condena a lo religioso, abriendo brecha a su 
comprensión como un elemento importante de la existencia de los pueblos y como una potencia vital de la Revolución.

El último texto, "Algunas reflexiones", escrito tras la caída de la URSS, en medio de la crisis en Cuba, analiza los elementos que llevan a la caída de un régimen socialista, el papel que juegan las ideologías en el fracaso de un régimen y cómo las modalidades de la moral operan frente a este problema.

\section{Sección II. Historia}

En un inicio, fue a través de la historia que FMH fue contrastando las nociones de la doctrina oficial con la realidad de su país. La historia fue el teatro en el que los combates de nuestro autor tuvieron más fuerza. De la reflexión teórica más general escribió muy poco, dejó pendientes trabajos que permitieran apropiarnos de ella, pero estos elementos quedaron presentes $y$ pueden rastrearse en materiales de estudio específico. En esta dimensión, la presente antología nos permite ubicar sus dos grandes líneas de trabajo, sobre el estudio de la historia y sobre la historia de Cuba. Sobre el estudio de la historia "Historia y marxismo", escrito en 1994, analiza las vulgarizaciones del marxismo en contraposición con los elementos clave en el trabajo del propio Karl Marx, así como las relaciones entre ideología, revolución e historia en Cuba. Mientras que en "Marx, el marxismo, Hobsbawm y nosotros", usando el concepto de presupuestos ideológicos, resalta los aportes del historiador británico para contrastarlos con sus relaciones y contradicciones con el proceso cubano. En "¿A dónde va el pasado?" expone su camino como historiador y da algunas pistas de las herramientas y el método que utiliza en el estudio de la historia.

El editorial del número 39 de Pensamiento Crítico, material invaluable para comprender la revolución del 30 en Cuba, nos permite un entendimiento de las posiciones que en la dimensión política subyacen a las distintas interpretaciones de la historia de aquel momento en Cuba -y en parte también de la actualidady cómo la historia tiene combates más allá del terreno de los hechos y datos, en especial cuando se lee desde la perspectiva del socialismo y la cuestión nacional.

Finalmente, este apartado se cierra con las "Visiones actuales de la historia de Cuba", donde realiza una reconstrucción panorámica de la ciencia histórica en Cuba tras el triunfo de la Revolución, el estado de los estudios históricos en relación con los problemas políticos de la actualidad y los compromisos y retos del historiador cubano hoy.

\section{Sobre la historia de Cuba}

En orden más o menos cronológico se recuperan distintos textos de FMH sobre la historia de ese país. Su enfoque parte de los subalternos, de las formas en que intervienen históricamente, tanto para adaptarse y sobrevivir a la historia dominante como para impugnarla.

Temas de la historia, en sus relaciones con las nociones de razas, clases y nación, dan forma a los cuatro primeros textos de esta subsección. Ellos permiten comprender cómo estas nociones operaron en la forja de la nación cubana.

Las revoluciones fueron para FMH un tema de suma importancia, en especial la revolución previa a la del 59, la de los años treinta (que él data desde 1930, mientras que por lo general se ubica su inicio en 1933). Le interesa su estudio porque afirma que ahí se forjó la noción de socialismo cubano, pero también porque a pesar de su radicalidad, la república burguesa se logró reacomodar y asimilar las luchas en una nueva República. La "Introducción" al libro La Revolución cubana del 30. Ensayos (Martínez Heredia, 2007) e "Ideas e ideologías en la Segunda República. La posición de Raúl Cepero Bonilla" se concentran en este gran tema.

La Revolución del 59, la que más conoció por su estudio y por ser un actor directo, fue sobre la que menos escribió. Sin embargo, los materiales que al respecto presentamos nos permiten ubicar, desde el ambiente ideológico, el acontecer de larga duración y algunos rasgos originales de su interpretación, que hacen que destaque del 
resto de los investigadores cubanos. "La noción de pueblo en La historia me absolverá", "El mundo ideológico cubano de 1959 a marzo de 1960" y "La fuerza del pueblo" son los textos que presentamos al respecto. Ellos nos brindan un conocimiento de aspectos centrales del desarrollo histórico de Cuba revolucionaria a la luz del presente.

\section{Sección III. América Latina}

FMH fue un gran conocedor de América Latina y escribió mucho sobre ella. Algunas veces informes sintéticos, de no más de dos cuartillas; otras veces ponencias breves para ser presentadas en 20 minutos; y en escasas ocasiones, textos más extensos para seminarios académicos. El conjunto de esta sección expresa una reflexión sobre América Latina basada en su experiencia de vida y en los problemas comunes que percibe en la región. Cuestiones como la identidad común y la existencia de una dimensión de proyecto frente al imperialismo y su complejo sistema de dominación cultural que, en la dimensión subjetiva, se sostiene principalmente- con cuatro rasgos: temor, fragmentación, indiferencia y resignación (Martínez Heredia, 2003). Reflexionó en torno a la opción liberadora y cómo esta debía articular en un plano central la lucha política con la cultura. Dice: "para lograr la liberación es impensable e ineficaz una política que no sea el instrumento de una grandiosa acción cultural" (Martínez Heredia, 2006: 23) en la que se deberá asumir al mismo tiempo y no como opuestas, la acción reformadora y la acción revolucionaria (Martínez Heredia: 2004: 313).

En esta sección los textos "Primeros pasos. Proyectos para el punto de partida", "Marxismo revolucionario en América Latina actual" e "Izquierda y reformismo en América Latina actual" se concentran en la defensa del socialismo como proyecto de liberación regional. Opuesto a considerar como caducas las fórmulas revolucionarias de los años sesenta, desde el acumulado cultural del presente, Fernando afirma que esas experiencias son básicas para los nuevos proyectos de liberación que tendrán que venir de los oprimidos, dominados y explotados (esos tres términos, integrantes de un sujeto popular, no son sinónimos) para asumir proyectos y poderes de transición socialista. "Siete retos para los jóvenes de América Latina" son incitaciones a asumir desde la juventud los desafíos que el sujeto popular debe hacer suyos en un esfuerzo liberador. Retos que aparecen en la dimensión personal porque en momentos de desarticulación y desarme, las posibilidades de remontar y salir avante recaen en las personas, con nombre y apellido. En el texto también puede apreciarse la relación de iguales que estableció FMH con las nuevas generaciones, a quienes incitó a no copiar recetas ni moldes. Finalmente, en cuanto a este texto, resaltamos que al ser los jóvenes los destinatarios, se puede comprender que la motivación principal estaba en que fueran las nuevas generaciones las que comprendieran su pensamiento. No esperaba que quienes nunca hicieron algo -lo decía así de brutalmente- cambien y asuman los desafíos que nunca quisieron asumir, deberán ser los nuevos los que superen a los viejos y abonen el camino del socialismo. El último texto que trabajó, "La revolución cubana en el siglo XXI", que sería el primer texto publicado post mortem, es publicado en esta sección, pues, aunque trata sobre Cuba, lo hace en el marco de una reflexión común sobre América Latina. Ahí, los desafíos, retos y problemas de Cuba son analizados desde la perspectiva latinoamericana y la interrelación de Cuba con la región para lograr un destino común de libertad y justicia.

\section{Sección IV. Socialismo}

La transición socialista fueel tema fundamental de FMH. Los contenidos expuestos hasta ahora -y los siguientes- se condensan y entretejen en esta cuestión. El concepto de transición socialista es el desarrollo teórico más acabado de $\mathrm{FMH}$ en torno a la revolución social. Es un concepto original del autor que permite pensar las bases propias de la lucha comunista desde América Latina a la luz de la experiencia cubana, pero no reducida a ella. Emplea el término para hacer énfasis en que es socialista del todo y es del todo una transición. No se está construyendo ni está ya construida (Martínez Heredia, 2013). La transición socialista, "época consistente en 
cambios profundos y sucesivos de las relaciones e instituciones sociales, y de los seres humanos, que se van cambiando a sí mismos mientras se van haciendo dueños de las relaciones sociales" (Martínez Heredia, 2013) es, ante todo, un poder político e ideológico, basado en la soberanía nacional y popular, y la justicia social, que consiste en un cambio cultural total.

Este concepto es un vínculo radical con el comunismo: comprende toda la época entre el capitalismo y el comunismo, que para ser tal, tiene que avanzar violentando una y otra vez las condiciones de reproducción de la economía, la política y la ideología, incluidas las creadas por ella misma, aunque de maneras muy diferentes a las que utilizó para derribar al capitalismo y la dominación imperialista (Martínez Heredia, 1988: 17). Por ende, la transición socialista es un concepto $\mathrm{y}$, al mismo tiempo, el desafío de hacerse, de darle vida a un proyecto revolucionario.

Hemos expuesto brevemente algunos elementos del concepto porque nos interesa insistir en su importancia y mostrar su relevancia en la obra de FMH y en el pensamiento social latinoamericano.

"Rectificación y profundización del socialismo en Cuba", "Desconexión, reinserción y socialismo en Cuba" y "Cuba: problemas de la liberación, el socialismo, la democracia" son algunos de los primeros textos en los que el autor desarrolló el concepto a fines de los ochenta e inicios de los noventa. No perdamos el contexto histórico y cómo la definición de socialismo es, entonces, una apuesta y no un elemento trasnochado del pasado, que no pierde actualidad porque el pensamiento dominante parezca haber triunfado. FMH no se conforma con estar acorde con el pensamiento en boga, sino que parte de la posibilidad de que las fuerzas sociales pongan el socialismo en el orden del día a partir de su acción subversiva, aun cuando esto parezca inviable.

"Dos socialismos. El caso de Antonio Guiteras" además de permitirnos conocer de entrada la importancia de Guiteras para el pensamiento cubano, expone la polémica en torno a dos socialismos en los momentos de su origen y defiende la vertiente socialista original cubana.

El texto "Independencia y socialismo en la América Nuestra", desde la dimensión latinoamericana, recalca la pertinencia del debate socialista en América Latina y cómo la transición socialista es un concepto que permite pensar el socialismo en Latinoamérica a partir de las condiciones establecidas, y no su postergación a un tiempo mejor. Frente al difícil escenario político, nos ayuda a pensar -por fuera de los ciclos y periodos- en nuevos horizontes epocales.

\section{Sección V. Cuba actual}

Seis textos, escritos en los años más recientes, 2016 y 2017 y solo uno de ellos en 2014, conforman esta sección. Los materiales fueron publicados previamente en distintos blogs, que en Cuba han sido el espacio privilegiado de lectura y debate político reciente. FMH muestra una apertura a usar estos espacios para debatir y lograr que las ideas lleguen a actores que, quizás solo a través de ellos, podrían acceder e interesarse por un pensamiento crítico. Los textos de $\mathrm{FMH}$ fueron recibidos, a través de los blogs, con entusiasmo por un sector activo -aunque no mayoritario- de jóvenes cubanos, que encontraron en ellos elementos de identidad rebelde con el proceso revolucionario cubano, herramientas para su defensa, y la invitación y apuesta a su radicalización desde un enfoque militante comprometido, distante a la búsqueda de la fama. "Problemas del socialismo cubano" es un texto profundo en el que se presenta la panorámica de desafíos para el momento actual. "O Cuba o Washington", "Los símbolos nacionales y la guerra cultural" y "Relaciones entre Cuba y Estados Unidos" se posicionan ante lo que parece una confusa y nueva forma de relaciones-agresiones de Estados Unidos para con Cuba. En ellos defiende la unidad de lo nacional con el socialismo, frente a quienes pudieran ubicar allí una diferencia o separación en pos de una apertura. Es tajante, y en esto su postura es polémica: cualquier cesión al capitalismo va por la vía de una posrevolución, 
de regreso al capitalismo y traición a la patria en un país -y región-donde la única posibilidad de ser soberanos se consigue con el socialismo. Y como el desafío es muy grande, en el texto "Las ciencias sociales cubanas: ¿el reino de todavía?" insiste en las enormes tareas del pensamiento en esta batalla que se libra, en la que el Che vuelve a ser fundamental. Por eso la sección la cierran las palabras de FMH en el taller sobre "El socialismo y el hombre en Cuba": para "Tomar conciencia y convertir la conciencia en acción organizada para transformar lo imposible en realidades, derribar todos los muros de la dominación burguesa y todas las prisiones del colonialismo, $\mathrm{y}$ desatar una sucesión interminable de revoluciones culturales", convertir al hombre común y corriente en un hombre nuevo (Martínez Heredia, 2017: 194-195). Algunos de estos textos están incluidos lo que sería el primer libro de FMH (2017) publicado post mortem, a fines de ese año, Cuba en la encrucijada. Libro que él había preparado para publicar a inicios del año, pero que tuvo retrasos en el proceso de edición e impresión. Ese libro y la selección presente se caracterizan por la defensa del proyecto socialista cubano, de su radicalización en una época en lo que domina en el ámbito intelectual, quizás no en los sectores populares, sea la defensa de una apertura que termina siendo resignación y adaptación a las reglas del mercado porque ya no son los mismos tiempos. Distante de un idealismo absurdo y consciente del tamaño del desafío revolucionario, las ideas de FMH pueden considerarse, en el ámbito del debate sobre Cuba actual, como las palabras necesarias, las que todos andaban buscando para asumir el desafío ambicioso de ir más allá del ámbito posible y tratar de ponerse a la altura que los revolucionarios de los cincuenta, pero también de los treinta y los del siglo pasado, tuvieron que alcanzar en la mayor de las Antillas para superar las condicionantes de su tiempo, que presentaban como absurdas, irracionales e idealistas las apuestas que se tornaron históricas.

Frank Josué Solar Cabrales, joven historiador cubano, en una entrevista con el autor de este prólogo, señaló que FMH asume hoy un rol similar al que asumió Raúl Roa para los años sesenta: una especie de puente histórico- generacional entre la revolución previa, en aquel caso la de los años treinta, y la actual, la que desató el Movimiento 26 de Julio en los años cincuenta. Hablar de FMH como conector de dos tiempos, así como lo fue Raúl Roa, no es un elogio, sino un desafío para quienes lo definen así, porque están planteando apropiarse del pensamiento de $\mathrm{FMH}$, pero sobre todo pasar a una acción que sea superior a sus circunstancias (Solar Cabrales, 2017).

\section{Sección VI. Determinación personal}

A primera vista esta sección podría considerarse una variopinta selección de relatos individuales. El conjunto muestra una reflexión en torno a un tema básico para el tema de las revoluciones: el del material humano que arranca los procesos que se convierten en históricos. Los procesos revolucionarios, como ya hemos planteado, alteran los marcos de lo concebido como posible en su tiempo. Se modifican los alcances, sentidos y valores de lo existente. $\mathrm{Su}$ estudio comúnmente deja de lado los elementos que operaron en las conductas humanas que detonaron la ruptura de un horizonte epocal. Se considera habitualmente que no fueron ellos, sino la historia; que no fueron sus actitudes, sino el medio; que fue la economía lo que como necesidad o determinación los impulsó a actuar de una u otra manera ¿Qué ocurre en las ciencias sociales que las revoluciones siempre aparecen como accidentes o excepciones? Al menos en las sociedades modernas la determinación personal resulta relevante, porque ella es el factor básico para desatar las revoluciones. La posible caída del capitalismo no se logrará porque este sea superado por las fuerzas que creó o porque se derrumbe a causa de su propio desarrollo. Contra el economicismo, Fernando pone en el centro que es la determinación de las personas la que forja, en la lucha de clases, los sucesos que alteran la dominación y, en todo caso, logra anular, frenar e instaurar otro tipo de sociedades. Es la experiencia de vida y el estar inmerso en un proceso revolucionario lo que va prefigurando ideas contrarias a los presupuestos de las ciencias sociales y a la escisión común entre lo objetivo y subjetivo. El Che Guevara, en "El socialismo y el hombre en Cuba", expone una 
idea importante la que pone el foco del problema en la actividad personal. Al referirse al inicio del proceso revolucionario cubano, dice: "el hombre era un factor fundamental. En él se confiaba, individualizado, específico, con nombre y apellido, y de su capacidad de acción dependía el triunfo o el fracaso del hecho encomendado" (Guevara, 1968: 81, 82). Ellos, los sujetos, fueron los que con su actividad alteraron lo objetivo e hicieron historia.

La posibilidad de ser del socialismo proviene de la voluntad y de la acción de las personas. Voluntad que es conformada por sentimientos y que alcanza a desarrollarse como conciencia y prefiguración de la sociedad que se quiere conquistar(MartínezHeredia, 2009:63).Entonces, lo que opera en la conducta revolucionaria de los individuos es la determinación personal, cuestión que anecdóticamente puede hacerse fácilmente comprensible, pero en lo analítico es más compleja. El autor cuenta que en una ocasión listó nueve dimensiones que conforman la determinación personal. Extravió el manuscrito y lamentablemente para las ciencias sociales, nunca volvió a desarrollar. Por carecer de un material teórico que pudiera comprender este concepto, los nueve textos de esta sección se convierten en pistas para su reconstrucción. Son muestras de cómo opera.

Una característica común a todos sus textos y que los diferencia de importantes corrientes del pensamiento crítico latinoamericano, es su rechazo a que la economía define -aun en última instancia, como suele rezar el dogma- la política y la historia. Para el autor, la economía no tiene ningún papel rector en la revolución, ni siquiera como última instancia (Martínez Heredia, 2010c: 187), pues lo que decide la economía es el funcionamiento de los regímenes de dominación (Martínez Heredia, 2009: 63). Es la praxis humana la hacedora de la historia. Lo subjetivo puede alterar la historia en la medida en que se hace movimiento histórico, esto es, en que las masas participan en ella. La centralidad está en la política. Los pueblos están forzados a tomar el poder. Por ende, el trabajo implica desarrollar esa centralidad. No apostar a que de las condiciones económicas devenga una opción política.
El primer texto, “¿Por qué Julio Antonio?", es el primer artículo que publicó el autor. Este, junto a los dos siguientes sobre Antonio Guiteras, analizan el papel de estas dos personalidades en la revolución del 30 . Su relación con la política después del 59 en Cuba es evidente. El texto "Recuerdo de Miguel Enríquez" y "Piñeiro", además de resultar una confidencia biográfica sobre el rol internacionalista de FMH y las relaciones con estos revolucionarios, es una evidencia de cómo se rescata la personalidad de ambos desde la lectura de las circunstancias en que vivieron y como las enfrentaron. Los detalles de las vidas se vuelven muestras de una identidad personal en la que los sentimientos, las actitudes, el estudio y la acción imprimieron su lugar en la historia.

El relato sobre Jacinto García -un personaje desconocido-precursor de la Revolución cubana del 59, y el de Hugo Chávez, revolucionario venezolano, resaltan cómo los esfuerzos personales y las motivaciones de ascenso social y superación humana, al encontrase con la dimensión política, hacen por su determinación personal que la vida se funda con la historia.

El texto sobre François Houtart y el titulado "Fe por Cuba" abordan el concepto de determinación personal desde las interrelaciones entre fe y revolución. En el primer caso desde la vida del intelectual belga y, en el segundo, desde una colectividad de cubanas y cubanos.

\section{Sección VII. Fidel y Che}

Esta sección pretende exponer la lectura de FMH sobre estos revolucionarios, y al hacerlo, mostrar la forma original en que son recuperados en su integralidad para pensar los problemas actuales. Resulta también una manera de darlos a conocer como unos de los más importantes exponentes del socialismo cubano, porque a pesar de ser las figuras más conocidas de la Revolución cubana de 1959, los lugares comunes que suelen llenar los vacíos y que muchas veces responden a formas en que se quiso reducir su radicalidad o trivializar. Sobre el Che se exponen dos materiales distintos entre sí. Uno es "La concepción del Che", un largo fragmento del 
libro premiado por Casa de las Américas en 1987, en la segunda versión que data de 2010 y que fue publicado con el título Las ideas y la batalla del Che (Martínez Heredia, 2010c). El otro es un texto que el autor elaboró para ser utilizado en labores de formación política por movimientos y organizaciones. Se pueden percibir dos formas distintas de abordaje, la primera con más detalle y citas, las segunda como material didáctico que resalta los rasgos del Che que el autor consideraba más urgentes a ser rescatados y comprendidos por quienes luchan.

Los textos sobre Fidel son recientes. Escribió muy poco sobre él, a pesar de ser un gran conocedor de su pensamiento y de ser partícipe de diversas labores que el comandante impulsó. No hablar de él, no permitir que se usara a Fidel como falacia de autoridad, no taparse con él y evitar revestir los argumentos con citas suyas, son algunas guías que, desde Cuba, conforman la ética martiana que asumió. Un texto es la ponencia que leyó a petición de los organizadores en el homenaje al cumpleaños 80 de Fidel y al aniversario 50 del desembarco del Granma, en el Palacio de Convenciones en La Habana, en diciembre de 2006; fue la única persona a la que se le pidió expusiera sobre el pensamiento de Fidel. El segundo, es la versión más acabada del material que publicó primero con el nombre "Fidel Vive" tras la muerte del máximo líder de la Revolución. Resultó ser el último texto que preparó para exponer fuera de Cuba y uno de los últimos en redactar. Permite conocer el pensamiento de Fidel, su actitud revolucionaria y las formas en que $\mathrm{FMH}$ pensó debía recuperarse su legado. En este apartado, con el estudio del pensamiento de estos dos personajes que, junto con Martí serían los grandes detonadores y guías de su reflexión, se condensan los temas tratados antes.

\section{Sección VIII. Sobre su vida}

FMH habló muy poco de su vida. En los primeros años del triunfo revolucionario se solía decir que quien hablaba de sí era porque quería algo -desde un elogio a un cargo-, y el optó por no hablar. Luego vino un largo periodo de silencio forzoso y en el momento en que volvió al debate lo fundamental no fue hablar de sí mismo. Hace solo unos pocos años empezó a contar algunas anécdotas, y con esta sección damos cuenta de esos relatos. Entre los propósitos de contar algunos episodios estaba su conciencia de que las historias de vida pueden ser alicientes para las nuevas generaciones en su búsqueda por asumir los desafíos de una nueva época. También, ante el interés que empezó a despertar en las nuevas generaciones la primera década de la Revolución, la búsqueda de la experiencia del grupo de la Calle K, la revista Pensamiento Crítico y la censura, Fernando decidió abundar en esas historias para contar y resaltar lo que consideraba debía ser lo fundamental a comprender. Sirve esta penúltima sección para que el lector conozca algunos episodios de la vida del autor en su propia voz y reflexione sobre ellos en relación con los demás textos de este libro.

\section{Sección IX. Entrevistas}

La entrevista como medio para difundir las ideas fue sumamente usada por FMH. Se trata de una herramienta para polemizar y llegar a auditorios diversos y a veces nuevos. Ligada en parte a la sección biográfica, en esta rescatamos las entrevistas de Néstor Kohan y Yohanka León en torno al Departamento de Filosofía, la revista Pensamiento Crítico y el marxismo en los años sesenta cubanos. La realizada por Emir Sader, en otro sentido, cubre algunos otros elementos biográficos. Incluimos un cuestionario sobre Lenin realizado en 1970 por el suplemento cultural El Caimán Barbudo del diario Juventud Rebelde y una entrevista realizada por Julio César Guanche en torno a su pensamiento político.

\section{IV}

"La vida de uno se va poblando también de soledades" escribió Fernando Martínez Heredia sobre el deceso de su amigo Alfredo Guevara (Martínez Heredia, 2015: 157). Meses más tarde, por el fallecimiento de Jaime Sarusky, confesó: "Mi madre nos enseñó a no tenerle miedo a la muerte, que es algo natural. A lo que temo realmente es a que muera nuestro tiempo". Pero añadió: "me sostiene la esperanza de que vendrán los nuevos y crearán un tiempo 
superior, en el que todos puedan sonreír y hacer bien cosas diferentes [... y] sientan el gozo de la vida como derecho de todos" (Martínez Heredia, 2015: 160). Resulta indudable que vivimos tiempos sombríos. El conjunto de metabolismos que garantizan la reproducción de la vida está en riesgo por la acción humana dominante. La lógica de reproducción de la vida social se mueve inconteniblemente hacia su autodestrucción.

En América Latina, la región más desigual del mundo, estos grandes peligros se juegan el destino -a veces de manera inmediata- de los humildes, los mayoritarios. $\mathrm{Y}$ en el escenario político, las fuerzas que enfrentan estos desafíos bajo la denominación de "progresistas" imprecisa en cuanto a sentido, pero correcta según el lenguaje de la realpolitik-, han sufrido fuertes reveses que se asoman como casi irremontables. Han sido orillados a reducir su horizonte de acción y planificación a una política del mal menor, en la que, cual advirtiera Antonio Gramsci, pueden sucumbir infinitamente, por inscribirse en un proceso de adaptación a un movimiento históricamente regresivo (Gramsci, 1999: 294-295).

El socialismo ha sido silenciado, se sostiene que es caduco, inoperante, que ha sido superado y que el capitalismo ha triunfado. Esto permea en todos los ámbitos en que domina el sistema, incluida la vida de los sectores populares e incluso más allá, en las mentes de quienes decididamente quieren enfrentar al sistema. ¿Por qué dejar de pensar en el socialismo? ¿Acaso la única opción ante lo que se vive es el pesimismo, la resignación y la derrota? ¿Por qué el pensamiento social no puede atreverse por voluntad de quienes lo elaboran a pensar de nueva cuenta en el socialismo? ¿Por qué olvidamos que los movimientos socialistas como el ruso o el cubano, que a posteriori fueron definidos como grandes hazañas históricasen su tiempo fueron acusados de idealistas y parecían enfrentarse a enormes imposibles?

Hace algunas décadas, en un tiempo que también se apreciaba sumamente oscuro, Ernst Bloch escribió: "En el suelo burgués [...] el cambio es de todo punto imposible, aun en el caso, -que no se da- de que efectivamente se deseara". En aquel tiempo, como en el nuestro -que es quizás el mismo si lo apreciamos desde las perspectivas de larga duración- la dimensión utópica parece cancelada. Lo que domina es una débil esperanza del advenimiento de una crisis del capitalismo, que por su dinámica propia y no la acción consciente contrapuesta a él, lleve a su muerte. "El interés burgués hasta quisiera incluir en su propio fracaso todo interés que se le oponga; para hacer desfallecer la nueva vida, convierte en aparentemente fundamental su propia agonía, en aparentemente ontológica" (Bloch, 2007: 27).

En ese sombrío contexto epocal, que no es distintoalqueFMHvivióy percibióen susúltimos días, es que su obra y su vida nos recuerdan el epígrafe de Bloch con que empezamos este texto: Pensar significa traspasar. En este caso, el pensamiento se fusiona con la acción y el traspaso toma concreción en la revolución socialista. Por eso, el nombre que toma esta antología, Pensar en tiempo de revolución, es la forma concreta en que la idea de Bloch, clave para comprender lo latente de la esperanza en momentos difíciles y de defenderla como posibilidad de realizarse, se expresa en nuestro autor y a su vez, la invitación que él nos hace para seguir en ese camino.

Fuera de tiempo y a su vez de este tiempo, el socialismo como ausencia reclama su actualidad no porque él mismo venga como producto de alguna crisis del sistema al que se enfrenta, no por necesidad, sino por voluntad. El socialismo como presencia se da por la decisión de crear algo nuevo y no por apelar a algo del pasado. Por las voluntades que puede despertar un movimiento histórico que impugne las dominaciones. Demanda ser discutido, ponerse en el espacio de los debates políticos y académicos que ya desde hace tiempo lo han expulsado. Porque siempre ha sido, sobre todo en Cuba, presente, vivencia, apuesta, proyecto que conjuga el presente, el pasado y el futuro. Fernando Martínez Heredia, un revolucionario comunista cubano, en la misma senda del Che, Fidel y aquellos que hace 100 años le abrieron, por vez primera, espacio real en esta tierra al socialismo, consagró su vida a la lucha por acabar con todas las dominaciones. Su 
obra nos abre muchas puertas para la reflexión y la acción liberadora y aquí tenemos un conjunto de materiales como documento vivo de lo que significa pensar en tiempo de revolución.

En la elaboración de esta Antología el trabajo de Esther Pérez fue fundamental. Agradezco su labor en la revisión y comentarios a este prólogo, el trabajo en la recuperación de los textos y las sabias opiniones compartidas.

\section{Bibliografía}

Bloch, E. 2007 El principio de esperanza (Madrid: Trotta) Trad. F. González Vicén.

Castro, F. 1958 “Carta a Celia Sánchez" en http:// www.fidelcastro.cu/es/imagen/cartaceliasanchez Acceso 3 de mayo de 2018.

Castro Ruz, F. 2001 [1953] La historia me absolverá. La Habana: Editorial de CienciasSociales.

Dorticós Torrado, O. 2013 “Lo más importante: que ustedes enseñen a pensar a los alumnos" en Bell Lara, J.; López García, D. L.; Caram León, T. (comps.). Documentos de la Revolución Cubana 1965. La Habana: Editorial de Ciencias Sociales.

Giulano, M. 1998 El caso Cea: Intelectuales e inquisidores en Cuba. ¿Perestroika en la isla? Miami: Ediciones Universal.

Gramsci, A. 1999. Cuadernos de la Cárcel. México: ERA T. V. Trad. A. M. Palos.

Guevara, E. 1968. El socialismo y el hombre en Cuba. Pensamiento Crítico 14.

Martínez Heredia, F. 1967. Presentación. Pensamiento Crítico 1.

Martínez Heredia, F. 1970. Editorial Pensamiento Crítico 39, edición especial, abril.

Martínez Heredia, F. 1988. Desafíos del socialismo cubano. La Habana: Centro de Estudios sobre América.
Martínez Heredia, F. 1989 Che, el socialismo y el comunismo. La Habana: Casa de las Américas.

Martínez Heredia, F. 2001a Corrimiento hacia el rojo. La Habana: Letras Cubanas /Instituto del Libro.

Martínez Heredia, F. 2001b Rosa Luxemburgo. Una rosa roja para el siglo XXI. La Habana: CIDCC Juan Marinello, Cátedra de Estudios Antonio Gramsci.

Martínez Heredia, F. 2003 Imperialismo, guerra y resistencia, Ponencia presentada en el III Foro Social Mundial, Porto Alegre, Brasil, enero.

Martínez Heredia, F. 2004. Primeros pasos. Proyectos para el punto de partida. Reforma ou Revolução? Para além do capitalismo neoliberal: concepções, atores e estratégias. San Pablo: Expressão Popular.

Martínez Heredia, F. 2006. Socialismo, liberación $y$ democracia. En el horno de los noventa (Melbourne / Nueva York: Ocean Sur).

Martínez Heredia, F. 2007. La Revolución cubana del 30. Ensayos. La Habana: Editorial de Ciencias Sociales / Ruth Casa editorial.

Martínez Heredia, F. 2009. Andando en la Historia. La Habana: ICIC Juan Marinello /Ruth Casa Editorial.

Martínez Heredia, F. 2010a. A viva voz. La Habana: Editorial de Ciencias Sociales.

Martínez Heredia, F. 2010b. El ejercicio de pensar. La Habana: Editorial de Ciencias Sociales / Ruth Casa Editorial) Segunda edición.

Martínez Heredia, F. 2010c. Las ideas y la batalla del Che. La Habana: Editorial de Ciencias Sociales / Ruth Casa Editorial.

Martínez Heredia, F. 2013 [2012] Socialismo. Ponencia presentada en el Seminario de Posgrado del Centro de Investigaciones 
Interdisciplinarias en Ciencias y Humanidades de la UNAM. México DF, 19 de septiembre.

Martínez Heredia, F. 2015. A la mitad del camino. La Habana: Editorial de Ciencias Sociales).

Martínez Heredia, F. 2016. Conversación de Fernando Martínez Heredia con Rafael Magdiel Sánchez Quiroz. La Habana, Cuba, 15 de octubre (mimeo).

Martínez Heredia, F. 2017. Cuba en la encrucijada. La Habana: Ed. Política / Ruth Casa Editorial.

Sánchez Quiroz, R. M. 2017. Rompiendo los límites de lo posible. El pensamiento crítico de Fernando Martínez Heredia, Tesis maestría. México: UNAM.

Solar Cabrales, F. J. 2017. Conversación de Frank Josué Solar Cabrales con Rafael Magdiel Sánchez Quiroz" La Habana, Cuba, 6 de junio (mimeo).

Tomado del Prólogo del libro, Rafael Magdiel Sánchez Quiroz, Pensar en tiempo de revolución, pp. 15-43. 